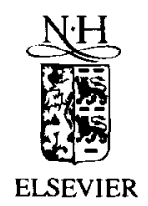

Human Movement Science 14 (1995) 391-400

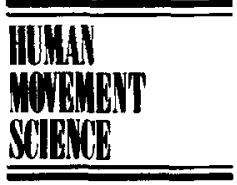

\title{
Coordination of multi-joint movements: An introduction to emerging views
}

\author{
A.J. van Soest ${ }^{a, b, *}$, G.P. van Galen ${ }^{c}$ \\ ${ }^{a}$ Institute of Fundamental and Clinical Human Movement Sciences, Amsterdam / Nijmegen, \\ The Netherlands \\ ${ }^{\mathrm{b}}$ Faculty of Human Movement Sciences, Free University, Van der Boechorststraat 9, 1081 BT Amsterdam, \\ The Netherlands \\ ${ }^{c}$ Nijmegen Institute for Cognition and Information, Nijmegen University, P.O. Box 9104, \\ 6500 HE Nijmegen, The Netherlands
}

\begin{abstract}
In this introductory paper, an overview is provided of the topics addressed in this special issue. These topics center around what is often referred to as Bernstein's problem. The first two topics both offer partial solutions to the indeterminacy problem. The first by identification of constraints acting on the neuro-musculo-skeletal system, in its interaction with the environment; the second by expanding the number of variables that are to be controlled. Regarding the first, it is argued that a distinction should be made between holonomic and nonholonomic constraints. Regarding the second, the necessity of independent control of in particular stiffness is a recurrent theme. The third and fourth topics concern choices to be made when modelling motor behavior. In particular, the level of detail at which the neuro-musculo-skeletal system is to be modelled in studies of coordination, and the merits of descriptive models offered by nonlinear dynamics are discussed. Apart from refining our models of the nervous system, the models of which are currently identified as a weak link, a major challenge for the coming years is concluded to lie in linking neuro-musculo-skeletal models to the behavioral models generated by nonlinear dynamics.
\end{abstract}

Early theorizing on the coordination of multi-joint movements was dominated by control theories usually referred to as "cognitive", "information processing", or "computational" theories. Well-known examples are

\footnotetext{
*Tel: + 3120444 8461. Fax: +31204445867.
} 
the schema theory of Schmidt (1975) and Rosenbaum's hierarchical editor model (Rosenbaum, 1991), but also recent explanations of classical findings such as Fitts' law as proposed by Bullock and Grossberg (1988) can be placed in this tradition. As noted by Beek et al. (1995), a problem with these theories is that many may be devised that account for observed behaviour, where it might be difficult to distinguish between these experimentally. Perhaps more importantly, the objection may be raised that in these theories, well-known structural properties of the human action system are not taken into account; for example, it is generally assumed in these theories that kinematic variables such as movement amplitude may be directly controlled. In contrast, Bernstein (1967) argued that top-level commands cannot fully specify what will happen kinematically. As internal state variables exist at different hierarchical levels in the neuro-musculoskeletal system, the kinematics of the movement must rather be conceptualized as emerging from the interaction between top-level commands, numerous internal states and kinetic as well as informational couplings to the environment (sec also Van Ingen Schenau et al., 1995). According to Bernstein, then, the central problem in the study of the coordination of multi-joint movements can be formulated thus: "how can we conceive of a theory of human action which explains that humans execute motor tasks in a highly consistent way, both intra- and inter-individually, notwithstanding the fact that the human action system contains a much larger number of neural, muscular and skeletal degrees of freedom than required by environment-related task goals".

How to resolve these "Bernsteinian" problems may be seen as the central issue addressed in this issue. In this context, it is important to note how Bernsteinian thinking has given new impulses to the motor programming paradigm. For example, in the contribution by Van Galen et al. it is shown how incorporation of the noise filtering properties of effectors may yield an alternative explanation for Fitts' law, an experimental finding that was previously explained on the basis of iterative control theory. Although reformulating the movement coordination problem in itself was an important step forward in that it led to new approaches within existing schools of thought, the main importance of Bernstein's work lies in the fact that it allows contributions from different disciplines to be juxtaposed and in some cases integrated. For example, this approach may help in bridging the gap between meaningful but poorly specified cognitive views of motor behaviour on the one hand, and dynamical and biomechanical principles on the other. Similarly, it may help bridge the gap between the muscle 
physiologist and the systems bioscientist, to use the terminology of Winters (1995). The contents of this special issue gives an indication to what extent such an integration of approaches is presently achieved.

In the remainder of this introductory paper, we will outline what we consider to be the main topics addressed in this special issue, and we will give a personal view on the way in which individual papers contribute to the discussion of these topics. This overview leads up to a final paragraph in which we will indicate, based on the contents of this special issue, which areas in the research of multi-joint movement coordination should be highlighted in the coming years.

\section{Imposing constraints reduces redundancy}

It may be argued that the apparent redundancy of the neuro-musculoskeletal system vis-a-vis task goals may not be as vast as previously thought because constraints reduce the number of degrees of freedom that are actually to be controlled. Note that this statement holds only if we use the term constraint in its strict sense: an algebraic relation between variables already in use. Thus, adding complexity to models (e.g., incorporating the gamma system into our actuator model, as proposed by Winters), even though it clearly has behavioural consequences, does not introduce constraints if it entails introduction of additional variables to be controlled (e.g., gamma activation and spindle feedback gain).

The topic of constraint-induced redundancy reduction is addressed by number of authors in this special issue. Gielen et al. (1995) provide an overview of constraints that have been proposed to be operating. Some of these are thought to be implemented neurally (e.g., task-specific relative activation of muscles; using only two rotational degrees of freedom to control subsystems with three rotational degrees of freedom); others are thought to arise from physical principles, in particular from musculo-skeletal mechanics (e.g., the tight coupling between joint rotations and translation of particular points on the skeleton; the tight relation between net joint torques and the force exerted on the environment; see also Van Ingen Schenau et al., 1995). Identification of such constraints is a prerequisite for proper understanding of the coordination of multi-joint movements. In our view, however, a clear distinction must be made between constraints that follow from physical principles ("holonomic constraints") and those that may be considered to be self-imposed ("non-holonomic constraints"). The 
rotation-translation coupling is an example of the first category; the use of two rotational degrees of freedom where three are available is an example of the latter category. Constraints of the first category make the system less redundant; self-imposed constraints must rather be viewed as part of the solution adopted by the system to overcome redundancy. It is an open question to what extent holonomic constraints are present in the nervous system. As argued by both Van Ingen Schenau et al. and Vaal et al., it is likely that the basic layout of the nervous system is genetically determined, but ample room exists for adaptation, in the terminology of Farmer (1990) both at the level of parameter dynamics (i.e., change of synaptic strengths) and at the level of graph dynamics (i.e., change of the architecture of networks).

Vaal et al. (1995) discuss neural and musculo-skeletal factors that have been suggested to be rate-limiting in the ontogenetic development of human locomotion, such as balance mechanisms and relative muscle force. There, a neuro-musculo-skeletal system is considered that is vastly redundant but that is yet not able to produce the behaviour of interest. Such factors may be considered to impose inequality constraints that need to be met before the qualitative change to independent locomotion can be achieved.

\section{Increasing task goal dimensionality reduces redundancy}

Many contributors to this issue bring up the issue of stability of movement and posture and its relation to muscle and/or joint stiffness and viscosity. It seems as though we are only currently realizing that the goal in reaching for a cup is not merely to get the hand to the right place. Clearly, any increase in the dimension of the task goal will make the system less redundant. This special issue seems to indicate that in particular stability of task execution may have to be considered as part of the task goal.

With respect to a description of behaviour and its stability properties under varying conditions, the theory of nonlinear dynamics as described by Beek et al., offers powerful concepts and tools. It is interesting to note that a dynamic model of very similar structure captures important aspects of behaviour at both kinematical and neurophysiological level. Although links between the phenomenological approach offered by nonlinear dynamics and underlying microdynamics are scarce at present (see below), this type 
of description does raise the question whether the goal of a task may include specification of the stability properties or whether the observed stability pattern is to be viewed as an emerging feature.

Van Galen et al. (1995) propose that in positioning movements, covariation of movement time and accuracy emerges from separate stiffness regulation of a noisy system. Winters (1995), confronted with the finding that stability of anti-gravity postures is hard to achieve on the basis of intrinsic musculo-skeletal dynamics, suggests an important refinement of our actuator model. Instead of viewing skeletal muscle as the actuator with alpha motoneuron activity being its input, he argues, we should include the muscle spindle system in the model of the actuator. This turns alpha motoneuron activity into a dependent variable, and shifts the controlled variables to a slightly higher level. It would seem that at least three variables (alpha motoneuronpool drive, gamma drive and spindle feedback gain) should now be controlled independently. Interestingly, Winters' proposal may come close to the structural basis of the control modalities proposed by Van Galen et al. Indeed, integration of well-described low-level neural circuitry into our actuator models will be an important step towards a generally applicable actuator model. As noted by Winters, such an approach obliterates the traditional controller-actuator separation, which is prevalent in robotics (see Gielen et al., 1995).

Vaal et al. (1995) carry the issue of stability one step further, when they suggest, in the context of the ontogenetic development of locomotion, that our control may be aimed at maintaining stable execution without taking out too much loans on intelligence. Thus, these authors appear to reject the tenet of Winters that optimization will be part of any attempt at modelling motor control. It must be realized, however, that stable execution with minimal cognitive load may well be reformulated into an optimality criterion.

Finally, we wish to point out that in equilibrium point theories (e.g., Feldman, 1966; Feldman, 1986; Bizzi et al., 1992) the stability of task execution has been a central point for quite some years. Yet, in their contributions both Gielen et al. and Van Ingen Schenau et al. raise a number of ohjections against these theories. In our view, the importance of equilibrium point theories lies in the fact that they focused attention on stability as an important property of any control strategy, and identified physiological processes (i.e., myotatic reflex loop; muscle's force-length relationship) that contribute to stability. 


\section{On the optimal level of detail in neuro-musculo-skeletal models}

From our earlier remarks concerning the physiological processes underlying equilibrium point models, it follows that forward dynamics multi-joint models to be used in studies of coordination should include a representation of those low-level processes that have consequences for control. Although this may seem obvious, to the present day significant efforts are (ill-)invested in developing forward dynamics models for studies of coordination where, for example, joint torque is used as the independent input. Clearly, the twofold question regarding the incorporation of more and more detail into our models is (1) where to draw the line, and (2) how to incorporate the relevant processes. At the level of skeletal dynamics, the contributors to this issue seem to agree that the skeletal linkage can be approximated by a number of rigid objects connected in fixed-axis joints, the behaviour of which is governed by Newtonian mechanics. It must be noted, though, that in particular the assumption of fixed rotation axes is not generally shared.

At the level of muscle physiology, some disagreement is clearly present between the multi-joint modeller (in the person of Winters) and the muscle physiologist (in the person of Huijing). Regarding the first question, Winters makes the important point that the relevance of particular physiological processes is task-dependent; thus, it is impossible to answer this question in general. According to Winters (1995), it is sound policy to construct general-purpose models at a somewhat more complex level, and to devise task-specific models by simplifying the general-purpose ones. Regarding the second question, Winters takes the pragmatic point of view that the simplest representation of a subsystem's behaviour is the best one. We tend to agree to this point of view, although we share one caveat with Huijing: the relation to the structural basis of the modelled phenomena must at all times be preserved.

Huijing (1995) provides an impressive overview of factors that complicate muscle's force-length characteristic. This overview is in sharp contrast with the view taken by the average muscle modeller, who typically assumes a fixed relation between length and force-per-unit-of-active-state. In Huijing's paper, a number of observations are reviewed that may indeed have important consequences for control. In particular, many of these observations are related to shifts of the force length relation to higher lengths. As a result, the muscle may effectively be operating below optimum length over a larger range of joint angles. This would simplify control as a muscle 
below optimum length behaves as a spring and is, thus, self-stabilizing, whereas a muscle above optimum length is unstable.

As stated by Huijing, virtually no information is available about the importance of these effects in vivo. Similarly Winters, based on his involvement in musculo-skeletal modelling of multi-joint movements, identifies a number of areas where muscle physiological data is urgently needed if we are to improve our muscle models. It appears that a lot is to be gained by a more intensive two-way collaboration between the system bioscientist and the muscle physiologist.

Regarding the neural subsystem, Van Ingen Schenau et al. (1995), inspired by Bernstein, provide some interesting ideas on the hierarchical organization of the nervous system, with tasks being delegated to a level as low as possible in the hierarchy. In particular, the point that "knowledge" of the effector system dynamics, in its interaction with the environment, is likely to be stored in neural networks, is quite appealing. However, it is difficult to envisage at present how these ideas are to be incorporated in a concretc model of motor behaviour. An important issue in modelling the nervous system concerns the level of abstraction at which the model is formulated. As noted by Gielen et al. (1995), a problem with the traditional artificial neural network (ANN) approaches is that ANN models may represent any input-output map. Alternatively, one might turn to more physiologically realistic models; apart from dimension problems, such models are hampered by the fact that neural layout and connection strengths will be impossible to validate. We tend to conclude that if Winters is right in stating that "an overall model is only as good as its weakest link", it is clear that in order to arrive at a general theory of coordination, the neural subsystem is the one that needs most of our attention.

\section{On the merits of descriptive models offered by nonlinear dynamics}

Beek et al. (1995) provide an introduction to the aims and methods of nonlinear dynamics. As argued there, it is interesting that the same dynamical principles capture the essentials of the behaviour of different action systems. Furthermore, nonlinear dynamics offers tools to arrive at a parsimonious description of both the behaviour at the level of state dynamics and of the stability properties of the behaviour as a function of (a small number of) control parameters. These tools are in principle applicable to different levels of analysis, e.g., kinematics, kinetics, neural activa- 
tion, although at present they are predominantly applied at the level of kinematics.

In some cases, phenomenological models have led the way to identification of the structural basis of the observed behaviour. In this respect, the Hodgkin-Huxley model for action potential generation and propagation is presented by Beek et al. as an example of a nonlinear dynamics model avant-la-lettre. A nice example indeed. In fact, it is argued by both van Ingen Schenau et al. and Winters that it is exactly this coupling to the structural basis that makes phenomenological models valuable. As noted by Van Ingen Schenau et al,, it is unfortunate that within the realm of coordination of multi-joint movements, the links between descriptive models and underlying microdynamics is rarely investigated, which casts doubt on the contribution of such models to our understanding of coordination. Those nonlinear dynamicists that consider coupling descriptive models to underlying microdynamics to be irrelevant, are heavily criticized by Van Ingen Schenau et al. In contrast, there is little reason to disagree with Beek et al., when they state that finding such links is one of the challenges to be confronted in future research. At present we can only hope that similarities and differences in nonlinear dynamics models and parameter values for different conditions and tasks will point the way to those processes at the level of microdynamics that are responsible for the observed behaviour.

\section{What's next?}

In our view, the study of coordination of multi-joint movements is aimed at unravelling the way in which humans cleverly exploit the intrinsic dynamics of their neuro-musculo-skeletal system in its interaction with the environment, resulting in adequate goal-directed behaviour. From the contents of this special issue, we draw a number of conclusions.

Firstly, our tools for description of movement seem to be quite adequate. Measurement of relevant variables at the levels of kinematics, kinetics and neurophysiology is routine procedure. Apart from the traditional descriptive studies, nonlinear dynamics offers new tools for rendering the dynamic structure of behavioural and neurophysiological data.

Secondly, our present models of the muscular and skeletal subsystems do allow us to study coordination of multi-joint models, albeit just so. Regarding skeletal system modelling, there is little discussion, although the assumption of fixed rotation axes may need critical evaluation. Regarding 
muscle dynamics, the situation is clearly more problematic: muscle physiology provides us with a description of the behavior of supramaximally activated muscle for a limited range of idealized length and force profiles. As noted by Winters, there are a number of areas (submaximal activation, eccentric contractions, "realistic" length and force inputs, fatigue) where data is scarce. Furthermore, structure-based parameter values for human muscles are not available for many muscle groups. In fact, it is remarkable that we do succeed to obtain reasonable results from simulations of multi-joint movements. Close cooperation between muscle physiologists and the modellers of multi-joint systems is required in order to arrive at a general actuator model.

Yet, in terms of the subsystems involved, the most problematic one is clearly the nervous system. Questions to be addressed in future research are: at what level of abstraction is the nervous system to be modelled? Is it viable to develop a general model of the nervous system, similar to the general actuator model that seems to be within reach, or are we bound to develop task-specific models with limited general value? Which neural subsystems need to be considered? At what level of abstraction is sensory information to be represented? How do cognitive concepts such as attention fit into our models of the nervous system? How important are neural processing times for stability? We can only hope that significant progress will be made on these issues in the years to come.

In parallel to refining our muscle models and investing in the development of models of the nervous system, the contents of this special issue gives rise to the feeling that the time has come to make a systematic attempt at an integration of current knowledge, from the level of muscle physiology to that of actor-environment behaviour. Let's leave our monodisciplinary constraints behind and accept the challenge to investigate if we can link forward dynamics neuro-musculo-skeletal models to the phenomenological models of nonlinear dynamics, taking both physical and self-imposed constraints fully into account!

\section{Acknowledgements}

We thank Peter Beek, Gerrit Jan van Ingen Schenau and Juliette Vaal for their helpful comments on an earlier version of this manuscript. 


\section{References}

Beek, P.J., C.E. Peper and D.F. Stegeman, 1995. Dynamical models of movement coordination. Human Movement Science 14, 573-608 (this issue).

Bernstein, N., 1967. The coordination and regulation of movements. New York: Pergamon Press.

Bizzi, E., N. Hogan, F.A. Mussa-Ivaldi and S. Giszter, 1992. Does the nervous system use equilibrium point control to guide single and multiple joint movements? Beh. Brain Sci. 15, 603-613.

Bullock, D. and S. Grossberg, 1988. Neural dynamics of planned arm movements: Emergent invariants and speed-accuracy properties during trajectory formation. Psychological Review 95, 49-90.

Farmer, J.D., 1990. A rosetta stone for connectionism. Physica D 42, 153-187.

Feldman, A.G., 1966. Functional tuning of the nervous system with control of movement or maintenance of a steady posture II. Controllable parameters of the muscle. Biophysics 11, 565-578.

Feldman, A.G., 1986. Once more on the equilibrium point hypothesis (lambda model) for motor control. J. Motor Beh. 18, 17-54.

Gielen, C.C.A.M., B.M. van Bolhuis and M. Theeuwen, 1995. On the control of biologically and kinematically redundant manipulators. Human Movement Science 14, 487-509 (this issue).

Huijing, P.A., 1995. Parameter interdependence and success of skeletal muscle modelling. Human Movement Science 14, 443-486 (this issue).

Rosenbaum, D.A., 1991. Human motor control. San Diego: Academic Press.

Schmidt, R.A., 1975. A schema theory of discrete motor skill learning. Psych. Rev. 83, 225-260.

Vaal, J., A.J. van Soest and B. Hopkins, 1995. Modelling the early development of bipedal locomotion: a multidisciplinary approach. Human Movement Science 14, 609-636 (this issue).

Van Galen, G.P. and W.P. de Jong, 1995. Fitts' law as the outcome of a dynamic noise filtering model of motor control. Human Movement Science 14, 539-571 (this issue).

Van Ingen Schenau, G.J., A.J. van Soest, F.J.M. Gabreels and M.W.I.M. Horstink, 1995. The control of multi-joint movements relies on detailed internal representations. Human Movement Science 14, $511-538$ (this issue).

Winters, J.M., 1995. How detailed should muscle models be to understand multi-joint movement coordination? Human Movement Science 14, 401-442 (this issue). 